\title{
The Role of Sex in Malaria-COVID19 Coinfection and Some Associated Factors in Rivers State, Nigeria
}

\author{
E. O. Onosakponome ${ }^{1}$ and M. N. Wogu $\mathbb{D}^{2}$ \\ ${ }^{1}$ Department of Medical Laboratory Science, PAMO University of Medical Sciences, Port Harcourt, Nigeria \\ ${ }^{2}$ Department of Animal and Environmental Biology, University of Port Harcourt, Nigeria \\ Correspondence should be addressed to M. N. Wogu; michael.wogu@uniport.edu.ng
}

Received 31 August 2020; Revised 15 November 2020; Accepted 23 November 2020; Published 7 December 2020

Academic Editor: María Eugenia López-Arellano

Copyright (C) 2020 E. O. Onosakponome and M. N. Wogu. This is an open access article distributed under the Creative Commons Attribution License, which permits unrestricted use, distribution, and reproduction in any medium, provided the original work is properly cited.

\begin{abstract}
Objectives. Data on the coinfection of malaria and COVID-19 is highly limited especially in Africa due to the novel nature of the pandemic COVID-19. Malaria and COVID-19 share striking similarities in their symptoms. A cross-sectional randomized study was conducted to investigate the role of sex in the coinfection of malaria and COVID-19 as well as some associated factors in Rivers State, Nigeria. Methods. Ethical approval was obtained from the Rivers State Health and Ethics Committee before the commencement of this study, and the study was conducted at the COVID-19 Treatment Center Medical Laboratory, Rivers State, Nigeria. Intravenous blood samples from three hundred randomly selected consenting study participants were examined for Plasmodium species using Giemsa microscopy, while pretested questionnaires were used to obtain data on sex, risk factors, and symptoms. All data generated were analyzed statistically using the Chi-square test with a $P<0.05$ value considered significant. Results. All study participants had Plasmodium species (100\% prevalence) with varying parasite loads, and $P$. falciparum was the only species observed. Study participants (irrespective of sex) with low and high parasitaemia had the highest and least prevalence, respectively $(P>0.05)$. Male study participants experienced more symptoms than females $(P>0.05$ ) except for sore throat which had an equal value among males and females. Travel history was the only risk factor that showed significant association with sex, and males had a higher value than females $(P<0.05)$. Conclusion. Malaria and COVID-19 are major public health issues in Nigeria; more researches on these diseases especially in epidemiology, pathology, diagnosis, treatment, and vaccine production are vital.
\end{abstract}

\section{Introduction}

Malaria is a major public health issue in Africa especially in the sub-Saharan region. It is the main cause of morbidities and mortalities in young children (0-5years) and pregnant women in many developing countries [1]. Malaria is mainly transmitted through the bites of infected female Anopheles mosquitoes. In late 2019, China experienced an outbreak of a new betacoronavirus with over 70,000 morbidities and 1,800 mortalities within two months. [2] The International Committee on Taxonomy of Viruses (ICTV) named the virus "SARS-CoV2" and the disease "COVID-19." [3, 4] COVID-19 is mainly transmitted to susceptible humans through infected nasal droplets which are released via coughing, talking, or sneezing. [5] There is very little data on malaria and COVID-19 co-infection glob- ally especially in African countries which experience both diseases with significant number of cases (morbidities and mortalities) daily. Sex (biological factor) and gender (behaviour and socio-cultural factors) are vital in understanding the dynamics of these diseases. Initial data from China and other countries globally have highlighted similar numbers of confirmed COVID-19 cases between men and women [6]. The present study was conducted to evaluate the role of sex (biological factor) in the co-infection of malaria and COVID-19 as well as some associated factors in Rivers State, Nigeria.

\section{Materials and Methods}

Before the commencement of this study, ethical clearance was obtained from the Rivers State Health and Ethics Com- 
TABLE 1: Prevalence of malaria and COVID-19 coinfection in relation to sex.

\begin{tabular}{lcccc}
\hline \multirow{2}{*}{ Sex } & \multirow{2}{*}{ Number examined } & \multicolumn{4}{c}{ Number infected (\%) } \\
& & LP & MP & HP \\
\hline Male & 179 & $89(49.7)$ & $51(28.5)$ & $39(21.8)$ \\
Female & 121 & $70(57.9)$ & $32(26.4)$ & $19(15.7)$ \\
Total & 300 & $159(53.0)$ & $83(27.7)$ & $58(19.3)$ \\
\hline
\end{tabular}

LP: low parasitaemia; MP: moderate parasitaemia; HP: high parasitaemia.

mittee. Study participants were three hundred randomly selected consenting laboratory confirmed COVID-19 patients. COVID-19 laboratory testing and confirmation was conducted prior to this study at the COVID-19 testing centres in the University of Port Harcourt Teaching Hospital (UPTH) and Rivers State University Teaching Hospital (RSUTH); both centres are located in Rivers State, Nigeria. Intravenous blood samples were collected from all study participants and examined for Plasmodium species using Giemsa microscopy according to standard laboratory methods [7]. Data on sex (male and female), symptoms experienced (fever, difficulty in breathing, cough/sneezing, headache, loss of smell or taste, running nose, and sore throat), and risk factors (travel history, nose mask usage, regular hand washing, and maintaining social distance) were also obtained from study participants. Malaria parasite load was graded into three groups: the high parasitaemia (HP), moderate parasitaemia (MP): and low parasitaemia (LP) groups. Data obtained were statistically analyzed by Chi-square test while Cramer's association coefficient and Fisher's exact were used to determine the goodness of fit for any significant Chisquare statistics. A $P$ value less than $0.05(P<0.05)$ was considered significant.

\section{Results}

All study participants had Plasmodium species (100\% prevalence) and $P$. falciparum was the only identified species. In Table 1, study participants with low parasitaemia (LP) had the highest prevalence of $49.7 \%$ and $57.9 \%$ in males and females, respectively, while those with high parasitaemia had the least prevalence of $21.8 \%$ and $15.7 \%$ in males and females, respectively $(P>0.05)$.

In Table 2, male study participants experienced more symptoms than females except for sore throat which had an equal value for males and females; all symptoms were not significantly associated with sex $(P>0.05)$.

Males had higher compliance to nose mask usage (58.9\%), regular hand washing (58.2\%), and maintaining social distance $(64.2 \%)$ as well as had higher recent travel history (91.2\%) (Table 3).

\section{Discussion}

All study participants had Plasmodium species and P. falciparum was the only species observed; some studies on malaria in Nigeria identified $P$. falciparum as the only malaria parasite [8-10]. Malaria is endemic in Rivers State,
TABLE 2: Relationship between symptoms and sex among study participants.

\begin{tabular}{|c|c|c|c|}
\hline \multirow{2}{*}{ Symptoms } & \multirow{2}{*}{ Number examined } & \multicolumn{2}{|c|}{$\operatorname{Sex}(\%)$} \\
\hline & & Male & Female \\
\hline \multicolumn{4}{|l|}{ Fever } \\
\hline Yes & 61 & $38(62.3)$ & $23(37.7)$ \\
\hline No & 239 & $141(59.0)$ & $98(41.0)$ \\
\hline \multicolumn{4}{|c|}{ Difficulty in breathing } \\
\hline Yes & 21 & $15(71.4)$ & $6(28.6)$ \\
\hline No & 279 & $164(58.8)$ & $115(41.2)$ \\
\hline \multicolumn{4}{|c|}{ Cough/sneezing } \\
\hline Yes & 53 & $32(60.4)$ & $21(39.6)$ \\
\hline No & 247 & $147(59.5)$ & $100(40.5)$ \\
\hline \multicolumn{4}{|l|}{ Headache } \\
\hline Yes & 57 & $35(61.4)$ & $22(38.6)$ \\
\hline No & 243 & $144(59.3)$ & $99(40.7)$ \\
\hline \multicolumn{4}{|c|}{ Loss of smell/taste } \\
\hline Yes & 131 & $73(55.7)$ & $58(44.3)$ \\
\hline No & 169 & $106(62.7)$ & $63(37.3)$ \\
\hline \multicolumn{4}{|c|}{ Running nose } \\
\hline Yes & 33 & $22(66.7)$ & $11(33.3)$ \\
\hline No & 267 & $157(58.8)$ & $110(41.2)$ \\
\hline \multicolumn{4}{|l|}{ Sore throat } \\
\hline Yes & 14 & $7(50.0)$ & $7(50.0)$ \\
\hline No & 286 & $172(60.1)$ & $114(39.9)$ \\
\hline
\end{tabular}

TABLE 3: Relationship between risk factors and sex among study participants.

\begin{tabular}{lccc}
\hline \multirow{2}{*}{ Risk factors } & \multirow{2}{*}{ Number examined } & \multicolumn{2}{c}{ Sex (\%) } \\
& & & Male \\
\hline Travel history & & $21(91.3)$ & $2(8.7)$ \\
$\quad$ Yes & 23 & $158(57.0)$ & $119(43.0)$ \\
$\quad$ No & 277 & & \\
Nose mask usage & & $56(58.9)$ & $39(41.1)$ \\
$\quad$ Yes & 95 & $123(60.0)$ & $82(40.0)$ \\
$\quad$ No & 205 & & \\
Hand washing & & $64(58.2)$ & $46(41.8)$ \\
$\quad$ Yes & 110 & $115(60.5)$ & $75(39.5)$ \\
$\quad$ No & 190 & & \\
Social distance & & $43(64.2)$ & $24(35.8)$ \\
$\quad$ Yes & 67 & $136(58.4)$ & $97(41.6)$ \\
$\quad$ No & 233 &
\end{tabular}

and it is unsurprising that all study participants had P. falciparum (despite varying parasite loads) but this also confirms that COVID-19 and malaria can be misdiagnosed in malariaendemic areas since both diseases share similar symptoms. More research is necessary to aid in adequately distinguishing COVID-19 from malaria (vice versa) especially in rural malaria-endemic areas which lack quality laboratories or testing centres and unavailable clinicians or health personnel. 
Males exhibited more symptoms than females in this study. A study conducted using 168 severe COVID-19 patients in Wuhan, China, reported that men were significantly prone to hospitalization and death as well as reduced likelihood of hospital discharge compared to women during the study [11]. In another research on SARS-CoV infection using mouse models, male mice had higher virus titres with more symptoms due to higher severe pulmonary damage from monocyte-macrophage infiltration and cytokine production [12]. More studies on co-infection of malaria and COVID-19 are needed to fully understand the role of sex (biological factor) and gender (behavioural and sociocultural factors) in relation to susceptibility, morbidities, and mortalities.

Males had higher recent travel history and were more compliant in undertaking necessary health precautions (washing hand frequently, regular wearing of nose/face masks, and maintaining adequate social or physical distance in public places). Travelling is a major factor in the epidemiology COVID-19 and malaria; travelling or migration is a significant factor in the epidemiology of diseases globally because it plays a key role in the existence, intensity, and dissemination of diseases within a population or area. Individuals (irrespective of their sex) should minimize their public movements (except when necessary), wash their hands frequently with soap and running water or use any alcoholbased hand sanitizer, properly wear nose masks or face shields especially in public or enclosed places, and always maintain a safe distance while in a public gathering with other individuals; these will reduce the spread of COVID19. The World Health Organization has emphasized that the response to the COVID-19 pandemic must utilize and strengthen existing infrastructure for addressing malaria and other infectious diseases globally [13]; therefore, results from this study will aid in formulating public health policies which will address the impact of COVID-19 epidemic on comorbidity, access, and control of malaria in relation to sex (biological factor).

\section{Conclusion}

Malaria and COVID-19 are major public health issues in Nigeria; more researches especially in epidemiology, co-morbidity, pathology, diagnosis, treatment, and vaccine production are vital to successfully curb the scourge of these diseases.

\section{Data Availability}

Data available on request (excluding names and sensitive information of study participants due to confidentiality/ethical reasons).

\section{Ethical Approval}

Ethical clearance was obtained from the Rivers State Health and Ethics Committee before the commencement of this study.

\section{Conflicts of Interest}

The authors declare that there is no competing interest in this study.

\section{Authors' Contributions}

All authors designed the study, performed the study analysis, and wrote the first draft of the manuscript.

\section{Acknowledgments}

The authors thank the laboratory staff, physicians, and study participants for their support.

\section{References}

[1] “CDC. Parasites-Malaria," https://www.cdc.gov/malaria/ malari_worldwide/impact.html.

[2] M. A. Shereen, S. Khan, A. Kazmi, N. Bashir, and R. Siddique, "COVID-19 infection: origin, transmission, and characteristics of human coronaviruses," Journal of Advanced Research, vol. 24, pp. 91-98, 2020.

[3] C. C. Lai, T. P. Shih, W. C. Ko, H. J. Tang, and P. R. Hsueh, "Severe acute respiratory syndrome coronavirus 2 (SARSCoV-2) and coronavirus disease-2019 (COVID-19): the epidemic and the challenges," International Journal of Antimicrobial Agents, vol. 55, no. 3, article 105924, 2020.

[4] J. Cui, F. Li, and Z. L. Shi, "Origin and evolution of pathogenic coronaviruses," Nature Reviews Microbiology, vol. 17, no. 3, pp. 181-192, 2019.

[5] C. D. C. Africa, "Coronavirus disease 2019 (COVID-19). Latest updates on the COVID-19 crises from Africa CDC," 2020.

[6] S. L. Klein, S. Dhakal, R. L. Ursin, S. Deshpande, K. Sandberg, and F. Mauvais-Jarvis, "Biological sex impacts COVID-19 outcomes," PLoS Pathogens, vol. 16, no. 6, article e1008570, 2020.

[7] M. Cheesbrough, part 1, District Laboratory Practice in Tropical Countries, Cambridge: Cambridge University Press, 2nd Edition edition, 2010.

[8] M. N. Wogu and F. O. Nduka, "Evaluating malaria prevalence using clinical diagnosis compared with microscopy and rapid diagnostic Tests in a tertiary healthcare facility in Rivers State, Nigeria," Journal of Tropical Medicine, vol. 2018, Article ID 3954717, 4 pages, 2018.

[9] A. AE and T. B, "Prevalence of malaria parasite among asymptomatic primary school children in Angiama community, Bayelsa State, Nigeria," Tropical Medicine and Surgery, vol. 4, no. 1, pp. 203-207, 2015.

[10] K. Pondei, L. Epidor, and N. Eno, "Prevalence of the malaria parasite in screened blood in a tertiary health centre in the malaria - endemic Niger-Delta region of Nigeria," Global Advance Research Journal of Microbiology, vol. 1, no. 11, pp. 188-193, 2012.

[11] Y. Meng, P. Wu, W. Lu et al., "Sex-specific clinical characteristics and prognosis of coronavirus disease-19 infection in Wuhan, China: a retrospective study of 168 severe patients," PLoS Pathogens, vol. 16, no. 4, article e1008520, 2020.

[12] R. Channappanavar, C. Fett, M. Mack, P. P. Ten Eyck, D. K. Meyerholz, and S. Perlman, "Sex-based differences in susceptibility to severe acute respiratory syndrome coronavirus 
infection," Journal of Immunology, vol. 198, no. 10, pp. 40464053, 2017.

[13] I. O. Ajayi, O. O. Ajumobi, and C. Falade, "Malaria and COVID-19: commonalities, intersections and implications for sustaining malaria control," The Pan African Medical Journal, vol. 37, no. 1, 2020. 